\title{
Generating optimal states for a homodyne Bell test
}

\author{
Sonja Daffer* and Peter L. Knight \\ Blackett Laboratory, Imperial College London, Prince Consort Road, London SW7 2BW, United Kingdom \\ (Received 14 January 2005; revised manuscript received 10 June 2005; published 1 September 2005)
}

\begin{abstract}
We present a scheme that produces a conditionally prepared state that can be used for a Bell test based on homodyne detection. The state is near optimal for Bell-inequality violations based on quadrature-phase homodyne measurements that use correlated photon-number states. The scheme utilizes a Gaussian entanglement distillation protocol and uses only beam splitters and photodetection to conditionally prepare a non-Gaussian state from a source of two-mode squeezed states with low squeezing parameter.
\end{abstract}

DOI: 10.1103/PhysRevA.72.034101

PACS number(s): 03.65.Ud, 03.65.Ta, 42.50.Xa, 42.50.Dv

Bell's theorem is regarded by some as one of the most profound discoveries of science in the 20th century. Not only does it provide a quantifiable measure of correlations stronger than any allowed classically, which is a key resource in many quantum-information processing applications, it also addresses fundamental questions in the foundations of quantum mechanics. In 1964, Bell quantified Bohm's version of the Einstein-Podolsky-Rosen (EPR) gedanken experiment by introducing an inequality that provides a test of local hidden variable (LHV) models [1]. A violation of Bell's inequality forces one to conclude that, contrary to the view held by EPR, quantum mechanics cannot be both local and real. In order to experimentally support this conclusion in a strict sense, a Bell test that is free from loopholes is required. Although it is still quite remarkable that such seemingly metaphysical questions can even be put to the test in the laboratory, a loophole-free Bell test has yet to be achieved.

For more than three decades, numerous experiments have confirmed the predictions of the quantum theory, thereby disproving local realistic models as providing a correct description of physical reality [2]. However, all experiments performed to date suffer from at least one of the two primary loopholes: the detection loophole and the locality loophole. The detection loophole arises due to low detector efficiencies that may not permit an adequate sampling of the ensemble space while the locality loophole suggests that component parts of the experimental apparatus that are not spacelike separated could influence each other. The majority of Bell tests have used optical systems to measure correlations, some achieving spacelike separations but still subjected to lowefficiency photodetectors (see, e.g., Ref. [3]). Correlations in the properties of entangled ions were shown to violate a Bell inequality using high-efficiency detectors, eliminating the detection loophole; however, the ions were not spacelike separated [4]. A major challenge that has yet to be achieved is to experimentally realize a single Bell test that closes these loopholes.

The ease with which optical setups address the locality loophole coupled with the currently achievable high efficiencies $(>0.95)$ of homodyne detectors makes Bell tests using quadrature-phase measurements good candidates for a

\footnotetext{
*Electronic address: s.daffer@imperial.ac.uk
}

loophole-free experiment. Furthermore, continuous quadrature amplitudes are the optical analog of position and momentum and more closely resemble the original state considered by EPR. Unlike photon counting experiments which deal with the microscopic resolution of a small number of photons, by mixing the signal with a strong field, homodyne measurements allow one to detect a macroscopic current [5].

In this Brief Report, we propose a test of Bell inequalities using homodyne detection on a conditional non-Gaussian "source" state, prepared using only passive optics and photon detection. Events are preselected-using event-ready detection one knows with certainty that the desired source state has been produced-requiring no post-processing. Photon detectors are only used in the preselection process and only affect the probability of successfully creating the source state whereas the actual correlation measurements are performed using high-efficiency homodyne detectors. The source is a correlated photon-number state that is near optimal for Bell tests using homodyne detection, opening the possibility of a conclusive, loophole-free test.

We consider a two-mode quantum state of light that can be written as

$$
|\Psi\rangle=\sum_{n=0}^{\infty} c_{n}|n, n\rangle,
$$

which is correlated in photon number $|n, n\rangle=|n\rangle_{A} \otimes|n\rangle_{B}$ for modes $A$ and $B$. For example, the two-mode squeezed state $\left|\psi_{\lambda}\right\rangle$ has coefficients given by $c_{n}=\lambda^{n} \sqrt{1-\lambda^{2}}$, where $\lambda$ $=\tanh (s)$ is determined by the squeezing parameter $s$ [6]. Such states are experimentally easy to generate; however, because they possess a Gaussian Wigner distribution in phase space, they are unsuitable for tests of Bell inequalities using quadrature-phase measurements as it is a requirement that the Wigner function possess negative regions [1]. Alternative, theoretically predicted two-mode quantum superposition states called circle states, also generated from vacuum fields through nondegenerate parametric oscillation, having coefficients given by $c_{n}=r^{2 n} / n ! \sqrt{I_{0}\left(2 r^{2}\right)}$, do exhibit a violation for quadrature-phase measurements with a maximum violation occurring for $r=1.12$ [7]. Unfortunately, unlike the two-mode squeezed states, circle states are difficult to realize experimentally. A recently proposed solution towards an experimentally realizable state of light 


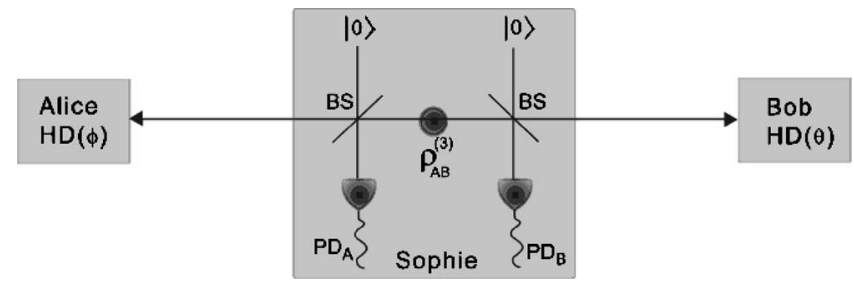

FIG. 1. A schematic diagram of the conditional homodyne detection for a Bell test. Sophie records successful preparation of the source state, denoted by $\left|\psi^{(3)}\right\rangle_{P S}$, while Alice and Bob perform homodyne measurements at their spacelike-separated locations. The three parties agree on which events to discard based on their measurement records.

that is suitable for a homodyne Bell test is the photonsubtracted two-mode squeezed state [8,9], having coefficients $c_{n}=\sqrt{\left(1-\lambda^{2}\right)^{3} /\left(1+\lambda^{2}\right)}(n+1) \lambda^{n}$, which utilizes nonGaussian operations on a Gaussian state. In this scheme, a photon is detected from each mode of a two-mode squeezed state and only the resulting conditional state is used for correlation measurements in the Bell test. While the two-mode squeezed state has a positive-everywhere Wigner function, the conditional state after photon subtraction does not. The nonclassicality of a single-mode photon subtracted squeezed state was studied in Ref. [10].

To date, all proposed states for a Bell test using quadrature-phase measurements are not optimal states, meaning that they do not produce the maximum possible violation of Bell inequalities. The scheme presented here produces a two-mode photon-entangled state that is near optimal, using only beam splitters and photon detection. The beam splitter may be described by the unitary operator [11]

$$
U_{a b}=T^{a^{\dagger} a} e^{-R^{*} b^{\dagger} a} e^{R b a^{\dagger}} T^{-b^{\dagger} b},
$$

which describes the mixing of two modes $a$ and $b$ at a beam splitter with transmissivity $T$ and reflectivity $R$. On-off photon detection is described by the positive operator-valued measure (POVM) of each detector, given by

$$
\Pi_{0}=|0\rangle\left\langle 0\left|, \quad \Pi_{1}=I-\right| 0\right\rangle\langle 0| .
$$

The on-off detectors distinguish between vacuum and the presence of any number of photons. The procedure is event ready, a term introduced by Bell, in the sense that one has a classical signal indicating whether a measurable system has been produced. The states demonstrating a violation of local realism presented here do not rely on the production of exotic states of light; in fact, only a parametric source generating states with a low squeezing parameter is required, making the procedure experimentally feasible with current technology. As depicted by Fig. 1, there are three parties involved: Alice, Bob, and Sophie. Sophie prepares the source states that are sent to Alice and Bob, who perform correlation measurements. We first describe the procedure Sophie uses to generate the source states, which is shown by the diagram in Fig. 2, and then discuss the measurements performed by Alice and Bob.

In the first step, two-mode squeezed states are mixed pairwise at unbalanced beam splitters followed by the non-

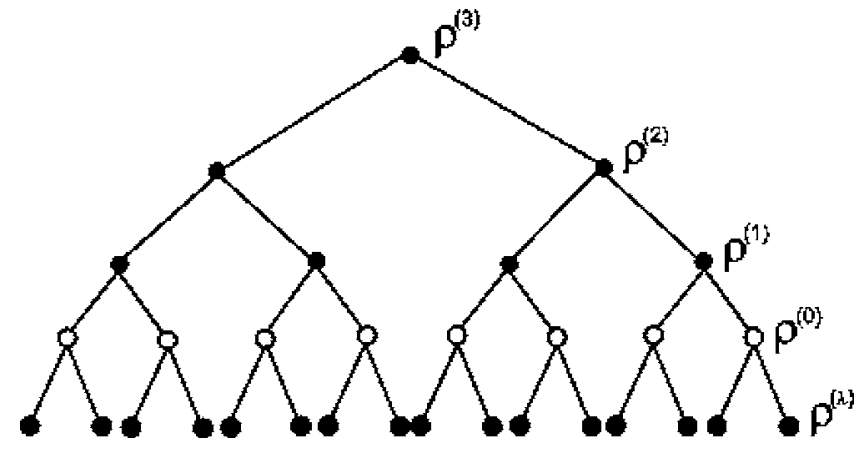

FIG. 2. A tree diagram of the conditional preparation of the state denoted by $\left|\psi^{(3)}\right\rangle$ from a finite supply of two-mode squeezed states $\left|\psi^{(\lambda)}\right\rangle$. Each circle represents the two-mode state as given on the right. The black (white) circles correspond to the Gaussian (nonGaussian) operations used to produce the states at each node.

Gaussian operation associated with the POVM element $\Pi_{1}$. Specifically, a non-Gaussian state is generated by

$$
\left(\Pi_{1, c} \otimes \Pi_{1, d}\right)\left(U_{a c} \otimes U_{b d}\right)\left|\psi_{\lambda}\right\rangle\left|\psi_{\lambda}\right\rangle,
$$

where $\left|\psi_{\lambda}\right\rangle$ denotes the two-mode squeezed state with $c_{n}=\lambda^{n} \sqrt{1-\lambda^{2}}$. For sufficiently small $\lambda$, the operator $\Pi_{1}$ describing the presence of photons at the detector approaches the rank-1 projection onto the single-photon number subspace $|1\rangle\langle 1|$, which is still a non-Gaussian operation. Under this condition, (un-normalized) states of the form

$$
\left|\psi^{(0)}\right\rangle=|0,0\rangle+\xi|1,1\rangle
$$

can be produced. That is, even though the output state of (4) will in general be a mixed state, when $\lambda \in[0,1)$ is very small, the resulting states can be made arbitrarily close in trace-norm to an entangled state with state vector given by Eq. (5), provided the appropriate choice of beam splitter transmittivity $|T(\lambda)|=\left|\xi-\sqrt{\xi^{2}+8 \lambda^{2}}\right| / 4 \lambda$ is used [12]. It should be emphasized that the state $\left|\psi^{(0)}\right\rangle$, having a Bell-state form, can be generated for arbitrary $\xi$.

It is interesting to note that the state given by Eq. (5) does not violate a Bell inequality for quadrature-phase measurements for any $\xi$, even when it has the form of a maximally entangled Bell state, as was shown in Ref. [13], in which a numerical study of the optimal coefficients for Eq. (1) was performed. For certain values of $\xi$, Eq. (5) describes a state that possesses a Wigner distribution that has negative regions, showing that negativity of the Wigner function is a necessary but not sufficient condition for a violation of Bell inequalities using quadrature-phase measurements.

The second step is to combine two copies of the state given by Eq. (5) pairwise and locally at 50:50 beam splitters described by the unitary operator of Eq. (2). Detectors that distinguish only between the absence and presence of photons are placed at the output port of each beam splitter, and when no photons are detected, the state is retained. The resulting un-normalized state is 


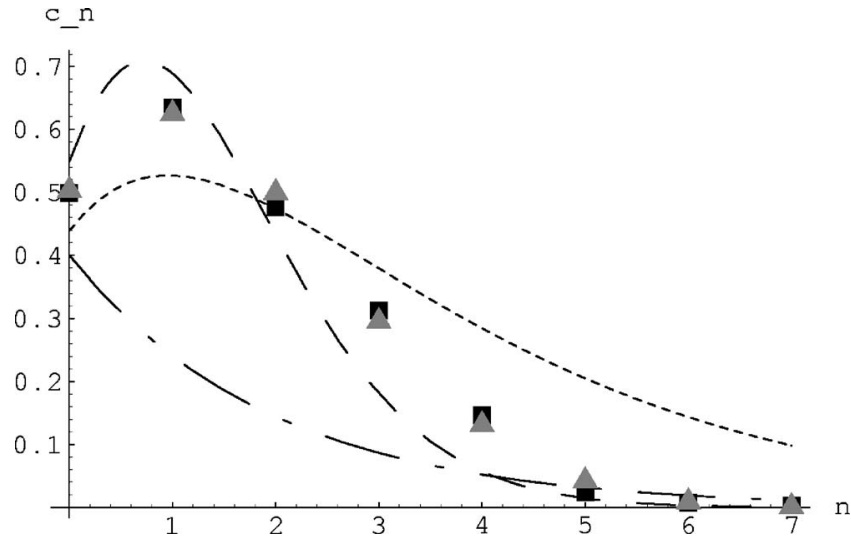

FIG. 3. The coefficients in Eq. (1) are plotted as a function of photon number $n$. The curves represent a photon-subtracted twomode squeezed state (small-dashed curve) for $\lambda=0.6$, the circle states (large-dashed curve) for $r=1.12$, and the two-mode squeezed state (dash-dotted curve) for $\lambda=0.6$. The optimal states are shown by squares and the source states $\left|\Psi^{(3)}\right\rangle_{P S}$ are shown by grey triangles for $\xi=0.71$.

$$
\left|\psi^{(i+1)}\right\rangle=\left\langle 0,0\left|U_{a c} \otimes U_{b d}\right| \psi^{(i)}\right\rangle\left|\psi^{(i)}\right\rangle=\sum_{n=0}^{\infty} c_{n}^{(i+1)}|n, n\rangle,
$$

where the coefficients are given by [14]

$$
c_{n}^{(i+1)}=2^{-n} \sum_{r=0}^{n}\left(\begin{array}{l}
n \\
r
\end{array}\right) c_{r}^{(i)} c_{n-r}^{(i)} .
$$

It is optimal to iterate this procedure 3 times so that Sophie prepares the state $\left|\psi^{(3)}\right\rangle$. Each iteration leads to a Gaussification of the initial state [12], which builds up correlated photon-number pairs in the sum of Eq. (1). Further iterations would Gaussify the state too much and destroy the nonlocal features for phase-space measurements.

The final step is to reduce the vacuum contribution by subtracting a photon from each mode of the state $\left|\psi^{(3)}\right\rangle$, obtaining a state proportional to $a b\left|\psi^{(3)}\right\rangle$. This is done by mixing each mode with vacuum at two beam splitters with low reflectivity. A very low reflectivity results in single-photon counts at each detector with a high probability when a detection event has occurred. Thus, the unitary operation describing the action of the beam splitter is expanded to second order in the reflectivity and the state is conditioned on the result $N=1$ at each detector. The final photon-subtracted state, given by

$$
\left|\psi^{(3)}\right\rangle_{P S}=\mathcal{N} \sum_{n=0}^{\infty}(n+1) c_{n+1}^{(3)}|n, n\rangle,
$$

where $\mathcal{N}$ is a normalization factor, is a near-optimal state for homodyne detection. Figure 3 compares the previously proposed states - the circle state and the photon-subtracted twomode squeezed state - with the near-optimal state $\left|\psi^{(3)}\right\rangle_{P S}$, as well as the numerically optimized state in Ref. [13]. The conditioning procedure alters the photon-number distribution of the input state and behaves similarly to entanglement distillation.
Although the procedure used to create the correlated photon source is probabilistic, with the success probability determined by the amount of two-mode squeezing and the transmittivity of the unbalanced beam splitters, it is event ready-Sophie has a record of when the source state was successfully prepared. Low-efficiency photon detectors used in the state preparation only affect the success probability and do not constitute a detection loophole. Each mode of the source state $\left|\psi^{(3)}\right\rangle_{P S}$ is distributed to a separate location where correlation measurements using high-efficiency homodyne detectors are performed by the two distant (spacelikeseparated) parties, Alice and Bob. Alice and Bob each mix their light modes with independent local oscillators (LO's) and randomly measure the relative phase between the beam and the LO's, taking into account the timing constraint that ensures fair sampling. Alice measures the rotated quadrature $x_{\theta}^{A}=x^{A} \cos \theta+p^{A} \sin \theta$ and Bob measures the rotated quadrature $x_{\phi}^{B}=x^{B} \cos \phi+p^{B} \sin \phi$. Correlations are considered for two choices of relative phase: $\theta_{1}$ or $\theta_{2}$ for Alice and $\phi_{1}$ or $\phi_{2}$ for Bob. Finally, Alice, Bob, and Sophie compare their experimental results to determine when the source state was successfully generated and which correlation measurements to use for the Bell inequalities.

Two types of Bell inequalities will be examined: the Clauser-Horne-Shimony-Holt (CHSH) and Clauser-Horne $(\mathrm{CH})$ inequalities [15]. To apply these inequalities, which are for dichotomous variables, the measurement outcomes for Alice and Bob are discretized by assigning the value +1 if $x \geqslant 0$ and -1 if $x<0$. Let $P_{++}^{A B}(\theta, \phi)$ denote the joint probability that Alice and Bob realize the value +1 upon measuring $\theta$ and $\phi$, respectively, and $P_{+}^{A}(\theta)$ denote the probability that Alice realizes the value +1 regardless of Bob's outcome, with similar notation for the remaining possible outcomes. From LHV theories, the following joint probability distribution can be derived:

$$
P_{i j}^{A B}(\theta, \phi)=\int \rho(\lambda) p_{i}^{A}(\theta, \lambda) p_{j}^{B}(\phi, \lambda) d \lambda,
$$

with $i, j= \pm$, by postulating the existence of hidden variables $\lambda$ and independence of outcomes for Alice and Bob. Quantum mechanically, the joint probability distribution is given by the Born rule $P\left(x_{\theta}^{A}, x_{\phi}^{B}\right)=\left|\left\langle x_{\theta}^{A}, x_{\phi}^{B} \mid \psi^{(3)}\right\rangle_{P S}\right|^{2}$. The probability for Alice and Bob to both obtain the value +1 is $P_{++}^{A B}(\theta, \phi)=\int_{0}^{\infty} \int_{0}^{\infty} P\left(x_{\theta}^{A}, x_{\phi}^{B}\right) d x_{\theta}^{A} d x_{\phi}^{B}$. The joint distribution is symmetric and a function of only the sum of the angles $\chi=\theta+\phi$, permitting the identification $P_{++}^{A B}(\theta, \phi)=P_{++}^{A B}(\chi)$ $=P_{++}^{A B}(-\chi)$ and $P_{++}^{A B}(\chi)=P_{--}^{A B}(\chi)$. The marginal distributions $P_{+}^{A}(\theta)=P_{+}^{B}(\phi)=1 / 2$ are independent of the angle. Given the probability distributions, the predictions of quantum theory can be tested with those of LHV theory.

First, we consider the Bell inequality of the CHSH type, which arises from linear combination of correlation functions having the form

$$
B=E\left(\theta_{1}, \phi_{1}\right)+E\left(\theta_{1}, \phi_{2}\right)+E\left(\theta_{2}, \phi_{1}\right)-E\left(\theta_{2}, \phi_{2}\right),
$$

where $E\left(\theta_{i}, \phi_{j}\right)$ is the correlation function for Alice measuring $\theta_{i}$ and Bob measuring $\phi_{j}$. These correlations are in turn determined by 


$$
E(\theta, \phi)=P_{++}^{A B}(\theta, \phi)+P_{--}^{A B}(\theta, \phi)-P_{+-}^{A B}(\theta, \phi)-P_{-+}^{A B}(\theta, \phi),
$$

obtained through the many measurements that infer the distributions $P_{i j}^{A B}(\theta, \phi)$. With the aid of the symmetry and angle factorization properties, the CHSH inequality takes the simple form $B=3 E(\chi)-E(3 \chi)$ with LHV models demanding that $|B| \leqslant 2$. The strongest violation of the inequality is obtained for the value $\chi=\pi / 4$; thus, a good choice of relative phases for Alice and Bob's measurements is $\theta_{1}=0, \theta_{2}=\pi / 2$, $\phi_{1}=-\pi / 4$, and $\phi_{2}=\pi / 4$. Using homodyne detection with optimal correlated photon-number states, the maximum achievable violation is 2.076 , whereas using the source states presented here, a Bell-inequality violation of $B=2.071$ is achievable.

Let us also consider the Clauser-Horne (strong) Bell inequality formed by the linear combination

$$
\frac{P_{++}^{A B}\left(\theta_{1}, \phi_{1}\right)-P_{++}^{A B}\left(\theta_{1}, \phi_{2}\right)+P_{++}^{A B}\left(\theta_{2}, \phi_{1}\right)+P_{++}^{A B}\left(\theta_{2}, \phi_{2}\right)}{P_{+}^{A}\left(\theta_{2}\right)+P_{+}^{B}\left(\phi_{1}\right)},
$$

denoted by $S$, for which local realism imposes the bound $|S| \leqslant 1$. Again, using the properties of the probability distributions, the simplification $S=3 P_{++}^{A B}(\chi)-P_{++}^{A B}(3 \chi)$ is possible. With the choice of the phases $\theta_{1}=0, \theta_{2}=\pi / 2, \phi_{1}=-\pi / 4$, and $\phi_{2}=\pi / 4$, a violation of $S=1.018$ is attainable given the states in Eq. (5) with parameter value $\xi=1 / \sqrt{2}$, which is quite close to the maximum value of 1.019 achieved by the numerical, optimal states in Ref. [13].

We have shown how it is possible to prepare a nearoptimal state for a Bell test that uses quadrature-phase homodyne measurements. Only very low squeezed states, passive optical elements, and photon detectors are required, making the procedure experimentally feasible at present. An initial state with a positive-everywhere Wigner function was succeeded by both non-Gaussian and Gaussian operations to prepare a state that exhibits a strong violation of both the $\mathrm{CHSH}$ and $\mathrm{CH}$ Bell inequalities. Efforts are currently being made towards an experimental realization of entanglement distillation for Gaussian states. The procedure presented here offers the opportunity for another possible experiment, as it utilizes a subset of an entanglement distillation procedure. Of course, any observed violation of a Bell inequality is sensitive to inefficiencies in the experiment that tend to deplete correlations. A full analysis involving dark counts and detection inefficiencies as addressed in Ref. [16] is necessary. Near-optimal states for homodyne detection may allow a larger window for experimental imperfections and offer the opportunity for a conclusive, loophole-free Bell test.

We thank Bill Munro and Stefan Scheel for useful comments and discussions. This work was supported by the U.S. National Science Foundation under Program No. NSF01154, by the U.K. Engineering and Physical Sciences Research Council, and the European Union.
[1] J. S. Bell, Physics (Long Island City, N.Y.) 1, 195 (1965); Speakable and Unspeakable in Quantum Mechanics (Cambridge University Press, Cambridge, England, 1987).

[2] A. Aspect, P. Grangier, and G. Roger, Phys. Rev. Lett. 49, 91 (1982); A. Aspect, J. Dalibard, and G. Roger, ibid. 49, 1804 (1982); Y. H. Shih and C. O. Alley, ibid. 61, 2921 (1988); Z. Y. Ou and L. Mandel, ibid. 61, 50 (1988); J. G. Rarity and P. R. Tapster, ibid. 64, 2495 (1990); J. Brendel, E. Mohler, and W. Martienssen, Europhys. Lett. 20, 575 (1992); P. G. Kwiat, A. M. Steinberg, and R. Y. Chiao, Phys. Rev. A 47, R2472 (1993); T. E. Kiess, Y. H. Shih, A. V. Sergienko, and C. O. Alley, Phys. Rev. Lett. 71, 3893 (1993); P. G. Kwiat, K. Mattle, H. Weinfurter, A. Zeilinger, A. V. Sergienko, and Y. Shih, ibid. 75, 4337 (1995); D. V. Strekalov, T. B. Pittman, A. V. Sergienko, Y. H. Shih, and P. G. Kwiat, Phys. Rev. A 54, R1 (1996); W. Tittel, J. Brendel, H. Zbinden, and N. Gisin, Phys. Rev. Lett. 81, 3563 (1998); J. C. Howell, A. Lamas-Linares, and D. Bouwmeester, ibid. 88, 030401 (2002); T. B. Pittman and J. D. Franson, ibid. 90, 240401 (2003); D. L. Moehring, M. J. Madsen, B. B. Blinov, and C. Monroe, ibid. 93, 090410 (2004).

[3] G. Weihs, T. Jennewein, C. Simon, H. Weinfurter, and A. Zeilinger, Phys. Rev. Lett. 81, 5039 (1998).

[4] M. A. Rowe, D. Kielpinski, V. Meyer, C. A. Sackett, W. M. Itano, C. Monroe, and D. J. Wineland, Nature (London) 409, 791 (2001).
[5] M. D. Reid, Quantum Semiclassic. Opt. 9, 489 (1997); Phys. Rev. Lett. 84, 2765 (2000).

[6] S. M. Barnett and P. L. Knight, J. Opt. Soc. Am. B 2, 467 (1985).

[7] K. Tara and G. Agarwal, Phys. Rev. A 50, 2870 (1994); A. Gilchrist, P. Deuar, and M. D. Reid, Phys. Rev. Lett. 80, 3169 (1998).

[8] H. Nha and H. J. Carmichael, Phys. Rev. Lett. 93, 020401 (2004).

[9] R. García-Patrón, J. Fiurášek, N. J. Cerf, J. Wenger, R. TualleBrouri, and Ph. Grangier, Phys. Rev. Lett. 93, 130409 (2004).

[10] M. S. Kim, E. Park, P. L. Knight, and H. Jeong, Phys. Rev. A 71, 043805 (2005).

[11] K. Wódkiewicz and J. H. Eberly, J. Opt. Soc. Am. B 2, 458 (1985).

[12] D. E. Browne, J. Eisert, S. Scheel, and M. B. Plenio, Phys. Rev. A 67, 062320 (2003).

[13] W. J. Munro, Phys. Rev. A 59, 4197 (1999).

[14] T. Opatrný, G. Kurizki, and D.-G. Welsch, Phys. Rev. A 61, 032302 (2000).

[15] J. F. Clauser, M. A. Horne, A. Shimony, and R. A. Holt, Phys. Rev. Lett. 23, 880 (1969); J. F. Clauser and M. A. Horne, Phys. Rev. D 10, 526 (1974).

[16] J. Eisert, D. E. Browne, S. Scheel, and M. B. Plenio, Ann. Phys. (N.Y.) 311, 431 (2004). 